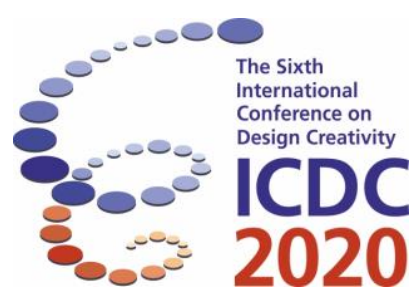

\title{
Enhancing creativity by demonstrating individual vulnerability to fixation
}

\author{
Maria A. Neroni and Nathan Crilly \\ Department of Engineering, University of Cambridge, UK
}

\begin{abstract}
Creative design behaviour can be inhibited by fixation and so reducing the risk of fixation is a focus of much design creativity research. Research in other fields indicates that an effective way of encouraging people to guard against a risk is to demonstrate that they, as individuals, are vulnerable to those risks. To study the effect of demonstrating individual vulnerability to fixation, we conducted an online experimental study using number and word tasks that are known to induce fixation. The first task was used to provide a 'demonstrated vulnerability' treatment (revealing the participants' own fixated behaviour to them) and to provide the explanation for a comparable 'asserted vulnerability' treatment (warning participants about general fixation effects). In the subsequent creative task, the 'demonstrated vulnerability' group outperformed those in the 'asserted vulnerability' group and also those in a control group.
\end{abstract}

Keywords: bias, demonstrated vulnerability, fixation, problem-solving, controlled experiment

\section{Introduction}

Creativity is often defined as the ability to produce ideas or solutions that are both novel (i.e. original, unexpected) and appropriate (i.e. relevant and useful to the problem at hand) (Cropley \& Cropley, 2010). Despite its recognised importance, creativity is often difficult to achieve, with people inadvertently 'fixating' on narrow interpretations of problems, a narrow range of solutions or a narrow set of problem-solving processes. Discussions of fixation in design have a long history (see review in Crilly, 2019), but the phenomenon was first experimentally studied thirty years ago by Jansson and Smith (1991) and has received continued research attention since then (see recent reviews by Sio, Kotovsky, \& Cagan, 2015; Vasconcelos \& Crilly, 2016).

One popular approach to reduce fixation in problem-solving is to give people (e.g. designers) warnings about it: warnings about the prevalence and effects of fixation. However, this approach has often proven to be ineffective. This might be explained by the observation that individuals believe themselves to not be at risk (as an individual) even if they believe such risks apply to people (more generally). An alternative approach, which has not been explored to date, is to demonstrate that people (e.g. designers) are individually vulnerable to fixation, demonstrations which might then motivate future corrective action. We conducted an experimental study to assess whether providing people with experiences that demonstrate their individual vulnerability to fixation would help them to resist later fixation episodes. In doing so, we aimed to provide knowledge about how we might reduce fixation effects and therefore suggest ways in which we might enhance creativity. 


\section{Literature review}

One common approach to reduce fixation effects is providing people with explicit warnings about the automatic repetition of inappropriate behaviours. However, the results of experimental studies using this approach are mixed. Some results are positive, such as Luchins' (1942) seminal work on the Einstellung effect (i.e. a fixating effect resulting from people's previous experience with a particular approach to solving problems). Luchins found that giving the instruction "don't be blind" to some of his experimental groups reduced the occurrence of the Einstellung effect by about 55 percent. Chrysikou and Weisberg (2005) also found that using de-fixation instructions (i.e. explicitly asking people to avoid using negative features from previously provided example solutions) eliminated the fixating effect of the example solutions in problem-solving. Similarly positive results are reported by Yilmaz, Seifert and Gonzalez (2010) and Howard, Maier, Onarheim and Friis-Olivarius (2013).

Whilst some researchers have found that warning about fixation is effective in reducing its occurrence, the opposite is also true. For example, Jansson and Smith (1991) did not find fixation to decrease when participants were instructed to avoid using the specific problematic features from the examples. Perttula and Sipila (2007) also clearly instructed participants not to reproduce the examples given in a creative task and arrived at similarly negative results (also see Vasconcelos, Neroni \& Crilly, 2018). However, perhaps most relevant to our interests here is the study by Smith, Ward and Schumacher (1993, experiment 3) who go further, warning one group of participants "We have found that examples like those you examined restrict people's creativity. Try NOT to restrict your ideas" (p. 843). They still found that this did not reduce the conformity effect observed in other groups who did not receive such a warning.

It seems that merely warning people about the risks of fixation is not a reliable way of motivating them to act against it (Perloff, 1987). As Mileti et al. (2004) state in their recommendations for how to communicate and educate people about public hazards, to concretely act against a risk, people must be convinced that that risk can happen to them. There are two useful bodies of prior work that we can consult for understanding how to demonstrate that individuals are susceptible to the risk of bias: (1) literature on the effect of revealing individual's unconscious social biases with the 'Implicit Association Test', and (2) literature on the effect of revealing individual's susceptibility to persuasion methods with a 'demonstrated vulnerability' treatment.

The 'Implicit Association Test' (IAT) is a set of computer-based trials were developed with the objective of revealing implicit biases in various matters of social cognition (most famously biases related to race, age and gender) (Greenwald, McGhee, \& Schwartz, 1998). An important feature of the IAT is its widely reported ability to reveal biases that might be unintentional, unknown, underestimated, denied or otherwise hidden by the participants (Greenwald et al., 1998). Because of this, it has been used for many years to reveal people's biases to them in a way that raises their awareness of the bias, their concern for its effects and their motivation to adopt strategies to address it (Devine, Forscher, Austin, \& Cox, 2012). The IAT has provided a platform for developing 'unconscious bias training programs' aimed at adjusting automatic patterns of thinking and reduce discriminatory behaviours through people's exposure (and subsequently reflection) on their own unconscious biases (e.g. Hannah \& Carpenter-Song, 2013). One way of framing this approach is that people are having their individual vulnerability to social bias demonstrated to them so that they can reflect on that bias and guard against it in the future.

Although the IAT is widely used, the idea of 'demonstrated vulnerability' is most explicit in Sagarin et al.'s (2002) work examining the effect of a treatment in which experimental participants are shown that they (as individuals) are susceptible to deception rather than just being told that they are (or that people are in general). This treatment was introduced in a study of how people can resist deceptive persuasive messages. Sagarin et al. (2002, Experiment 3) compared the behaviour of different experimental groups, including those given an 'asserted vulnerability' treatment (asked to reflect on the possibility that they had been fooled by an unethical advert) and those given a 'demonstrated vulnerability treatment' (provided with evidence that they had been fooled by an unethical advert). When those participants responded to subsequent adverts, it was found that, the asserted vulnerability treatment was not enough, participants had to have their vulnerability demonstrated for an effective resistance to be developed. 


\section{Method}

In the present study, we sought to develop and test the efficacy of a 'demonstrated vulnerability' intervention for fixation. We adopted the general experimental structure of Sagarin et al.'s (2002) study, with three groups: demonstrated vulnerability, asserted vulnerability and control. We used two fixation tasks: for our conditioning of the independent variable we used a number task (adapted from Bugelski and Huff's (1962) revision of Luchins' (1942) 'three jars' task) and for measuring the effect on our dependent variable we used a word task (Cowen and Thompson's (1951) 'alphabet maze' task). These relatively 'closed' problem-solving tasks are more restrictive than the 'open' design tasks typically used in design fixation research. With respect to the independent variable, this allowed us to reliably show participants that they had solved the problem in one specific and well-defined way, rather than another (superior) way. With respect to the dependant variable, it allowed us to objectively measure fixation. We hypothesised that experiencing the 'demonstrated vulnerability' intervention with the three jars task would make participants in that group more resistant to fixation in the later alphabet maze task compared to participants in the asserted vulnerability group (who had been only warned about the risk of fixation) and in the control group (who had not previously received any intervention related to fixation).

\subsection{Participants}

One-hundred sixty-eight participants (30 female) were recruited into the study by responding to a posted advertisement published on the online platform Amazon Mechanical Turk. We restricted the recruitment to participants who were native English speakers, aged between 18 and 30 years and studying at university. Their average age was 26.42 years $(\mathrm{SD}=3.39)$. Before starting the study, participants read an on-screen Information Sheet and gave their consent to participate. Participants received a small honorarium (5 USD) in return for their participation. The study procedures were approved by the local ethical review committee.

\subsection{Materials and procedure}

At the beginning of the study, participants were randomly assigned to one of three experimental groups: demonstrated vulnerability, asserted vulnerability, and control. Participants in all groups were individually involved in two sessions: the tasks in Session 1 varied according to the experimental group; the tasks in Session 2 were common to all the groups. For both sessions and all the tasks, the instructions were presented on a computer and all activities were conducted on a computer (for methodological considerations see Neroni, Vasconcelos, \& Crilly, 2017). Each participant accessed the study by entering the Amazon Mechanical Turk platform and then following a link leading to the Qualtrics survey software. An initial introduction explained to the participants that they would complete a series of tasks involving numbers and letters and answer some questions. Then, participants were introduced to Session 1.

\subsubsection{Session 1: demonstrated vulnerability group}

For the demonstrated vulnerability group, Session 1 included a computerised version of the three jars task. In each problem, participants viewed images of three jars marked 'A', 'B', and ' $C$ ' with numbers indicating their capacities, and a target vessel with a number indicating the volume of liquid that should be transferred into it. The capacities of the three jars as well as the required target volume varied across problems. The task included of two sets of problems: five 'set problems' that could all be solved by the same complex sequence of pouring operations (i.e. B-A-2C) and one 'test problem' that could be solved using the same method but a simpler (and easier) solution was also possible (i.e. $\mathrm{A}+\mathrm{C}$ ). For each problem, participants were required to find the simplest solution (i.e. the one that required the fewest pouring operations).

Following the completion of the three jars task, participants were invited to examine their response to the 'test problem' and asked whether or not this response indicated that they had noticed the alternative, simpler solution. Depending on their response to this question, participants were given a slightly different explanation of fixation: participants who had been unable to find the simpler solution 
to the 'test problem' were informed about the possibility of this alternative solution and were told that their inability to find it when working on that problem was probably due to a 'fixation effect'; participants who had been able to find the simpler solution to the 'test problem' were congratulated on their performance and informed that many people are unable to identify the alternative, simpler solution due to a 'fixation effect'. Following this individual feedback, all the participants were provided with a deeper explanation of fixation and were given some examples of real-life fixation behaviours. Finally, participants were invited to keep in mind what they had learnt in Session 1 when working on the following tasks.

\subsubsection{Session 1: asserted vulnerability group}

For the asserted vulnerability group, Session 1 included an online lecture on 'fixation', including an introduction to the concept, a description of the 'three jars task' as a way to study fixation with typical results, and some examples of real-life fixation behaviours. To ensure that participants had carefully read the text and understood the concept of fixation, their knowledge was then tested with a set of five multiple choices questions. The amount and type of information about fixation that participants in this group received (in the text) was the same as in the demonstrated vulnerability group (i.e. the same task was used to introduce and explain the concept of fixation, using the same number and same type of fixation examples). If participants answered the questions incorrectly they were informed of this and allowed to try again until the correct answers were given. They were allowed to move to Session 2 only if they had answered all the questions correctly. Finally, participants were invited to keep in mind what they had learnt in Session 1 when working on the following tasks (just as with the demonstrated vulnerability group).

\subsubsection{Session 1: control group}

For the control group, Session 1 included an arithmetic task. The arithmetic task was a modified version of the three jars task. Participants were presented with a set of arithmetic problems each involving a combination of jars having different capacities. The participants were required to calculate a target volume of liquid by applying the formula ' $\mathrm{B}-\mathrm{A}-2 \mathrm{C}$ '. In each problem, participants viewed images of three jars marked ' $\mathrm{A}$ ', 'B', and ' $\mathrm{C}$ ' with numbers indicating their capacities, and a target vessel with a question mark indicating the volume of liquid that should be calculated. The capacities of the three jars as well as the required target volume varied across problems.

\subsubsection{Session 2: all groups}

After completing Session 1, all the participants moved to Session 2. For all groups, Session 2 included a computerised version of the alphabet maze task. Participants viewed a series of $6 \times 6$ grids with each square of the grid containing a letter of the alphabet. For each grid, participants were required to identify a continuous and meaningful path of words by moving from the top right corner to the bottom left corner of the grid.

The task consisted of three sets of problems: (i) six 'set problems' which could only be solved using the same long path through the maze (i.e. a ten-letter solution), (ii) one 'test problem' which could be solved via the previous long path but also via an alternative, shorter path (i.e. a six-letter solution), and (iii) one 'extinction problem' that could only be solved with the alternative shorter path (in this last case, the long path did not result in a meaningful phrase).

For each problem, participants were required to try to find the shortest solution (i.e. the one that used the fewest letters). As with the three jars and the arithmetic task, participants were first given a 'practice problem'. The 'practice problem' consisted of an example grid that could be solved with a path that was different from those required to solve the three sets of previously described problems (to avoid participants applying the path that worked in the practice problem to solve the following problems).

At the end of Session 2, all the participants were asked to answer some demographic questions, indicating their sex, age, level of education and field of study. Participants in the demonstrated vulnerability group and in the asserted vulnerability group were also asked to provide the following information: (i) how often they thought they had previously experienced fixation (i.e. once per minute, 
once per hour, once per day, once per week, once per month, once per year); (ii) an example of a reallife fixation episode that they had experienced (to ensure that they had correctly understood the concept of fixation and to link what they had learnt in the study to their everyday life); (iii) state if they believed that knowing about their own (for the demonstrated vulnerability group) or other people's (for the asserted vulnerability group) vulnerability to fixation could have a positive effect on helping them to avoid fixation in the future. The total testing time was about 40 minutes per participant (see Figure 1 for a summary of the activities completed by the participants in each group).

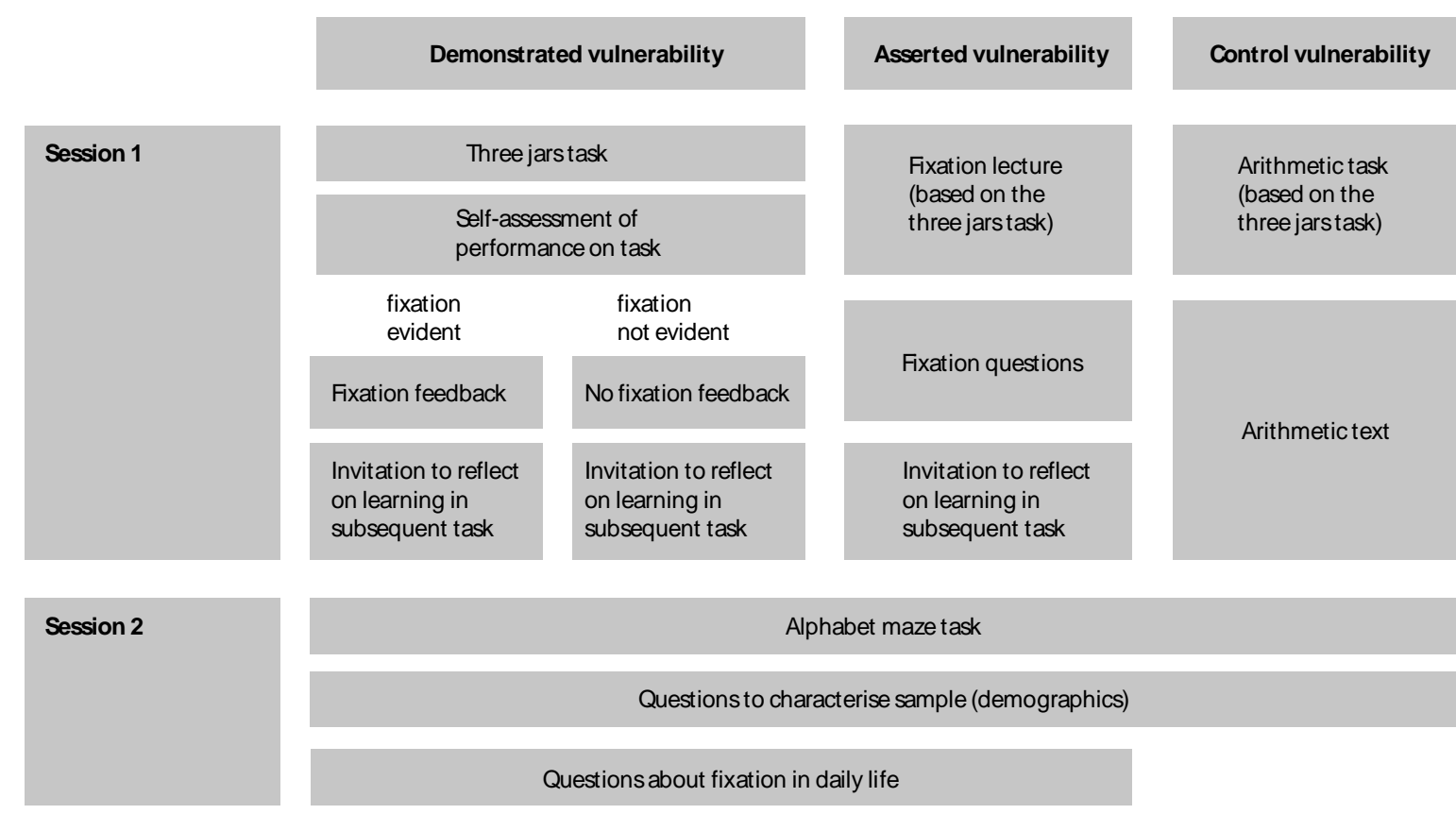

Figure 1. Tasks, activities and follow-up questions completed by the participants in the three groups.

\section{Results}

Fifty-nine participants were removed from the analysis because they did not complete the activities properly. The final sample therefore consisted of 109 participants (demonstrated vulnerability $=36$; asserted vulnerability $=35$; control $=38$ ).

As Session 1 was simply used to manipulate the participants' knowledge of and experience of fixation (the independent variable), participants' behaviour was analysed only in relation to their performance in Session 2 (the dependent variable).

The results revealed that more participants in the asserted vulnerability group (94.29\%) and in the control group (92.10\%) persisted in applying the long path that worked in the 'set problems' to solve the 'test problem' compared to the participants in the demonstrated vulnerability group $(72.22 \%), \chi^{2}(2)$ $=8.97, p=.01, \varphi=.29$ (see Figure 2). No significant difference was observed between the asserted vulnerability group and the control group, $\chi^{2}(1)=.14, p=.71, \varphi=.04$. This result was unaffected by removing those (three) participants who did not become fixated or those (two) who incorrectly evaluated their own performance with respect to fixation. Participants in the three groups did not significantly differ in relation to their response times on the 'test problem' (demonstrated vulnerability group: $\mathrm{M}=14.08, \mathrm{SD}=6.58$; asserted vulnerability group: $\mathrm{M}=21.27, \mathrm{SD}=21.47$; control group: $\mathrm{M}$ $=17.54, \mathrm{SD}=10.13), F(2,106)=2.32, p=.10, \eta_{\mathrm{p}}^{2}=.04$. 


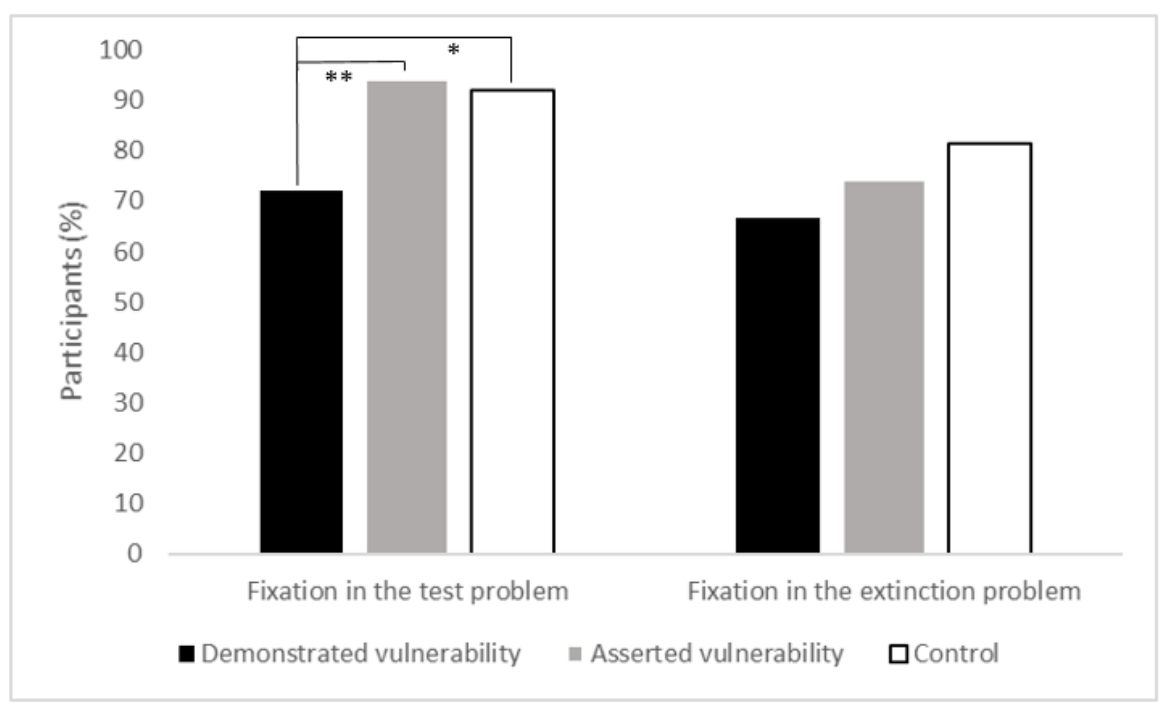

Figure 2. Percentage of participants in each group who persisted in applying the (inefficient) long path to solve the 'test problem' in the letter maze task (left), and failed to identify a meaningful solution to the 'extinction problem' (right).

All the participants (except three participants in the control group) attempted the 'extinction problem'. However, most participants failed to provide a meaningful solution to this problem ( 77 out of 109 , or $70.64 \%$ of the participants, see Figure 2). There was no significant difference between the three groups in relation to the number of participants who failed to give a meaningful solution to the "extinction problem', $\chi^{2}(2)=1.64, p=.44, \varphi=.12$. However, among those who were able to identify a meaningful solution to the 'extinction problem' $(33.33 \%$ of the participants in the demonstrated vulnerability group, $25.71 \%$ of the participants in the asserted vulnerability group, and $18.42 \%$ participants in the control group), participants in the demonstrated vulnerability group were significantly faster in correctly identifying that solution $(M=10.88, S D=9.78)$ compared to the participants in the asserted vulnerability group $(M=29.64, S D=18.68)$ and in the control group $(M=29.41, S D=27.65), F(2$, $25)=3.54, p=.04, \eta_{\mathrm{p}}{ }^{2}=.22$. No significant difference was observed between the asserted vulnerability group and the control group, $t(14)=0.02, p=.98, d=.009$.

The results of the participants' answers to the follow-up questions revealed that participants in the demonstrated vulnerability group and in the asserted vulnerability group did not differ in relation to their beliefs about the frequency that they experienced fixation in their lives (most common answer in both groups was 'once per day', $36.11 \%$ of the responses in the demonstrated vulnerability group and $28.57 \%$ of the responses in the asserted vulnerability group), $\chi^{2}(2)=5.57, p=.47, \varphi=.28$.

Finally, more participants in the demonstrated vulnerability group (34 out of 36, or $94.44 \%$ ) believed that knowing about their own/other people's vulnerability to fixation could have a positive effect on helping them to avoid fixation in the future, compared to the participants in the asserted vulnerability group (22 out of 35 , or $62.86 \%), \chi^{2}(1)=10.63, p=.001, \varphi=.39$.

\section{Discussion}

The participants in our demonstrated vulnerability group outperformed the participants in the asserted vulnerability group and the control group. This supported our hypothesis that providing people with experiences that demonstrate their individual vulnerability would give rise to a significantly stronger tendency to resist later fixation episodes than simply asserting that people in general are vulnerable to fixation. The effectiveness of demonstrating vulnerability to fixation has not previously been shown in experimental studies. The results do, however, fit with previous qualitative research reporting that expert designers recognise previous fixation episodes and reflect on those in future work to reduce the occurrence and influence of fixation (Crilly, 2015). Our research objective here has been to explore how such experiences and self-analysis might be accelerated. Our use of a computer-based task provides a basis for considering how a demonstrated vulnerability treatment might be implemented in 
real-life contexts of creative training or development. In considering computational support for creative education, Burleson (2005) tells us that "it is possible to accelerate the pace of learning through exposure to difficult circumstances that may arise less frequently than in real world situations. This will inevitably accelerate the rate of failure and, if motivation is sustained, the rate of learning as well” (p. 445).

Despite the clear results, our study has a number of specific features which should be considered when generalising to other settings or when planning future work. We used two brief closed-ended tasks, both of which provided objective measures of solution quality (number of arithmetic operations; number of letters used). Whilst these tasks were methodologically convenient, how do they relate to design activities? Of course, they might be directly analogous to solving the closed problems which form an essential component of much technical design work, such as software coding and structural detailing. It might also be that demonstrating fixation vulnerability on a closed problem (such as the three jars task) improves creative performance on more open-ended problems. That is something we don't know, but it would be an important question for future work to explore. Either way, conventional design fixation research protocols suffer from various problems of subjectivity (Neroni, Vasconcelos \& Crilly, 2017) and have unproven ecological validity (Crilly, 2019), so exploring and testing alternative approaches is important.

Our two tasks were performed in quick succession. Would we see the same effects with a substantial time delay between them? The longer-term effects of design fixation interventions are seldom studied or reported, but there is some evidence that creativity training can have long-lasting effects (see review in Scott, Leritz, \& Mumford, 2004: p. 375). Although interventions to improve design performance 'in the wild' might realistically target short-term performance improvements (e.g. see Linsey, Wessen, \& Ziemer, 2016), such interventions are likely to be considered most valuable if they have longer-term effects. Although our participants in the demonstrated vulnerability group reported that they believed the positive effect would be long-lasting (more so than in the asserted vulnerability group), we still don't know what kind of intervention would really be required for that to be achieved. If interventions of this kind only have short-term effects, then they might need to be performed repeatedly (for example, at the start of each ideation session) in order to maintain improved levels of creative performance over the longer term (for example, throughout a multi-month project).

\section{Conclusion}

Previous efforts to reduce fixation effects have often involved providing people with training or warnings about fixation and encouraged them to guard against exhibiting fixation in later tasks. There are various interrelated reasons to suspect that these approaches might be ineffective and that demonstrating vulnerability might be more effective. However, there is another popular approach: providing creativity tools and techniques. Our results are relevant to this approach also because such tools and techniques might be more effective if combined with demonstrating individual vulnerability to fixation. If we are interested in reducing the effects of people's problem-solving biases in creative activities then we should account for the biases that they might have toward such biases. We should understand that they might underestimate the degree to which creative tools and techniques are relevant to their own abilities and practices. We've shown that demonstrating individual vulnerability to fixation can improve performance in subsequent tasks. It might also improve engagement with creativity tools and techniques, leading to them being applied more effectively. The best interventions might require a combination of multiple approaches, with a demonstration of individual vulnerability to fixation motivating people to guard against it by whatever means are available to them.

\section{Acknowledgements}

This work was supported by the UK's Engineering and Physical Sciences Research Council (EP/K008196/1). Research data supporting this publication are available from https://doi.org/10.17863/CAM.51070.

\section{References}

Bugelski, B. R., \& Huff, E. M. (1962). A note on increasing the efficiency of Luchins' mental sets. The American journal of psychology, 75(4), 665-667. 
Burleson, W. (2005). Developing creativity, motivation, and self-actualization with learning systems. International Journal of Human-Computer Studies, 63(4), 436-451.

Chrysikou, E. G., \& Weisberg, R. W. (2005). Following the wrong footsteps: fixation effects of pictorial examples in a design problem-solving task. Journal of Experimental Psychology: Learning, Memory, and Cognition, 31(5), 1134.

Cowen, E. L., \& Thompson, G. G. (1951). Problem solving rigidity and personality structure. The Journal of Abnormal and Social Psychology, 46(2), 165-176.

Crilly, N. (2015). Fixation and creativity in concept development: The attitudes and practices of expert designers. Design Studies, 38, 54-91.

Crilly, N. (2019). Methodological diversity and theoretical integration: Research in design fixation as an example of fixation in research design? Design Studies, 65, 78-106.

Cropley, D., \& Cropley, A. (2010). Functional creativity: "Products" and the generation of effective novelty. In J. C. Kaufman \& R. J. Sternberg (Eds.), The Cambridge handbook of creativity (pp. 301-317). New York, NY, US: Cambridge University Press.

Devine, P. G., Forscher, P. S., Austin, A. J., \& Cox, W. T. (2012). Long-term reduction in implicit race bias: A prejudice habit-breaking intervention. Journal of experimental social psychology, 48(6), 1267-1278.

Greenwald, A. G., McGhee, D. E., \& Schwartz, J. L. (1998). Measuring individual differences in implicit cognition: the implicit association test. Journal of personality and social psychology, 74(6), 1464.

Hannah, S. D., \& Carpenter-Song, E. (2013). Patrolling your blind spots: introspection and public catharsis in a medical school faculty development course to reduce unconscious bias in medicine. Culture, Medicine, and Psychiatry, 37(2), 314-339.

Howard, T. J., Maier, A., Onarheim, B., \& Friis-Olivarius, M. (2013). Overcoming design fixation through education and creativity methods. In 19th International Conference on Engineering Design (pp. 139-148).

Jansson, D. G., \& Smith, S. M. (1991). Design fixation. Design Studies, 12(1), pp. 3- 11.

Linsey, J. S., Wessen, R., \& Ziemer, J. (2016). Observations of a highly innovative group-Directions for future research. The Fourth International Conference on Design Creativity, 1-9. Atlanta, GA.

Luchins, A. S. (1942). Mechanization in problem solving-the effect of Einstellung. Psychological Monographs, 54, No. 6: 1-95.

Mileti, D., Nathe, S., Gori, P., Greene, M., \& Lemersal, E. (2004). Public hazards communication and education: the state of the art. University of Colorado: Natural Hazards Centre.

Neroni, M. A., Vasconcelos, L. A., \& Crilly, N. (2017). Computer-Based "Mental Set" Tasks: An Alternative Approach to Studying Design Fixation. Journal of Mechanical Design, 139(7), 071102-071102-071110.

Perloff, L. S. (1987). Social comparison and illusions of invulnerability to negative life events. In Coping with negative life events (pp. 217-242). Springer, Boston, MA.

Perttula, M., \& Sipilä, P. (2007). The idea exposure paradigm in design idea generation. Journal of Engineering Design, 18(1), pp. 93-102.

Sagarin, B. J., Cialdini, R. B., Rice, W. E., \& Serna, S. B. (2002). Dispelling the illusion of invulnerability: The motivations and mechanisms of resistance to persuasion. Journal of personality and social psychology, 83(3), 526.

Scott, G., Leritz, L. E., \& Mumford, M. D. (2004). The effectiveness of creativity training: A quantitative review. Creativity Research Journal, 16(4), 361-388.

Sio, U. N., Kotovsky, K., \& Cagan, J. (2015). Fixation or inspiration? A meta-analytic review of the role of examples on design processes. Design Studies, 39, 70-99.

Smith, S. M., Ward, T. B., \& Schumacher, J. S. (1993). Constraining effects of examples in a creative generation task. Memory \& Cognition, 21(6), 837-845.

Stone, J., \& Cooper, J. (2001). A self-standards model of cognitive dissonance. Journal of Experimental Social Psychology, 37(3), 228-243.

Vasconcelos, L. A., \& Crilly, N. (2016). Inspiration and fixation: Questions, methods, findings, and challenges. Design Studies, 42, 1-32.

Vasconcelos, L. A., Neroni, M. A., \& Crilly, N. (2018). The effect of explicit instructions in idea generation studies. AI EDAM, 32(3), 308-320.

Yilmaz, S., Seifert, C. M., \& Gonzalez, R. (2010). Cognitive heuristics in design: Instructional strategies to increase creativity in idea generation. AI EDAM, 24(3), 335-355. 